\title{
Coagulase-Negative Staphylococci in Blood Cultures: The Clinician's Dilemma
}

Coagulase-negative staphylococci have made quantum leaps in recent years toward respectability as bona fide pathogens. They are among the most common organisms isolated from infections of prosthetic cardiac valves, cerebrospinal shunts, peritoneal dialysis catheters, and indwelling intravenous catheters. However, the majority of coagulase-negative staphylococci recovered from clinical specimens are still of questionable significance. Kirchhoff and Sheagren, in this issue of Infection Control, provide data that coagulase-negative staphylococci, although the most common organism recovered from blood culture bottles in their hospital, were rarely responsible for bacteremia. ${ }^{1}$ In fact, by their criteria, only 33 of 527 patients $(6.3 \%)$ having one or more blood culture bottles growing coagulase-negative staphylococci had a true bacteremia with this organism. These data are very similar to those generated by Weinstein et al who found that only $6 \%$ of 163 routine blood cultures growing coagulase-negative staphylococci came from patients who truly had the organism growing in their blood. ${ }^{2}$ Both studies used criteria that excluded the most obviously "contaminated" cultures and relied upon chart review to decide the validity of positive blood cultures among the rest. The results of these studies are important because they emphasize the need for increasing cooperation between clinicians and the clinical microbiology laboratory over the processing and reporting of routine cultures. Since coagulase-negative staphylococci are the most common culture contaminant they are a great source of wasted effort and

From the Departments of Medicine and Microbiology/Immunology, Medical College of Virginia, Virginia Commonwealth University, Richmond, Virginia.

Address reprint requests to Gordon L. Archer, MD, Box 49, MCV Siation, Richmond, VA 32398-000I. expense for the clinical microbiology laboratory. On the other hand, organisms growing in blood represent one of the best indicators to the clinician of true infection and, because of the high prevalence of indwelling vascular devices in hospitalized patients, coagulase-negative staphylococci have a unique access to the blood. There are several actions that can be taken that may help to resolve this quandary.

First, it is important to identify methods that the clinical laboratory can use to separate those cultures that have a high probability of being contaminants from those more likely to represent true bacteremia. The following four situations are highly suggestive of contamination: a single positive blood culture followed by multiple negative cultures; only one of two simultaneously-drawn blood cultures growing the organism; two positive cultures from the same patient separated in time by multiple negative cultures; and growth in only the aerobic or anaerobic bottle of a simultaneously-inoculated pair. The latter situation, as emphasized by Kirchhoff and Sheagren, is a particularly good tip-off that contamination occurred during inoculation. Most species of coagulase-negative staphylococci are facultative anaerobes and should grow equally well in both bottles of an aerobic-anaerobic pair if the bottles are inoculated with equal volumes of blood.

When a blood culture has been identified as having a high probability of contamination with a coagulase-negative staphylococcus, the clinical laboratory should include a message to the doctor on the reporting slip or computer screen and proceed no further with susceptibility testing unless notified by the physician. This would save the laboratory an inordinate amount of time and resources that would normally be wasted doing susceptibility tests on clinically meaningless isolates. However, it is important that clinicians be given the opportunity to decide for 
themselves the relevance of blood culture isolates that are likely to be contaminants. Some patients (ie, patients with prosthetic valve endocarditis) may have infrequent positive cultures separated by negative ones; it may not be possible to obtain more than a single culture in other patients (eg, pediatric patients); and some species of coagulase-negative staphylococci (eg, S. saprophyticus) do not grow well anaerobically.

How can the significance of blood cultures that infrequently grow coagulase-negative staphylococci be ascertained? One way is by identifying the same unique phenotypic markers on two different isolates. Unusual phenotypic characters should be unlikely to occur on two contaminants isolated from blood cultures at different times. However, most hospital-acquired coagulase-negative staphylococci have similar biotypes (species $S$. epidermidis), phage types (untypable), and antibiograms (multiply antibiotic-resistant). Combinations of all three phenotypic markers have been used but performance of all of these tests can be extremely labor-intensive. ${ }^{3}$ Genotypic markers have been sought as well. Plasmid typing has been useful in confirming the identity of multiple isolates from patients at high risk of infection but it requires technical expertise that at present would make it difficult to perform routinely. ${ }^{4}$ A larger panel of biochemical reactions for biotyping would be helpful in the identification of unusual phenotypic markers as would gene probes for the detection of unique DNA sequences. The availability of sensitive, specific, relatively inexpensive, and easy-to-perform typing systems for coagulasenegative staphylococci would be immensely helpful to the clinical microbiology laboratory clinicians faced with multiple positive cultures of unknown significance, and epidemiologists trying to contain the spread of nosocomial infections caused by these organisms.

Secondly, although the number of coagulase-negative staphylococci isolated from blood culture bottles that are the cause of true bacteremia is a small percentage of the total number recovered, true bacteremia occurs in an impressively large number of patients. Ponce de Leon and Wenzel found that coagulase-negative staphylococci were one of the most important causes of true nosocomial bacteremia in their hospital over a 7 -year study period." Their incidence of nosocomial bacteremia, 7 per 10,000 admissions, is almost identical to the figure of true bacteremia that can be calculated from the study of Kirchhoff and Sheagren. Furthermore, if only the number of patients with three or more blood cultures positive for any organism is considered, coagulase-negative staphylococci were second only to $S$. aureus as a cause of bacteremia during the 26-month period of Kirchhoff and Sheagren's study. The presence of the organism in blood is, therefore, an important marker for nosocomial infection and of particular concern to the hospital epidemiologist. Coordination between the hospital epidemiologist and the clinical microbiology laboratory can identify high-risk areas for nosocomial coagulase-negative staphylococcal bacteremias (ie, intensive care units and oncology wards). Routine susceptibility testing of isolates from patients in these areas may be indicated. Computer programs linking the clinical microbiology laboratory and the hospital epidemiology unit would further facilitate these decisions.

Finally, all individuals responsible for drawing blood for cultures must continually be reminded of the importance of proper technique and the economic and patient care implications of contaminated cultures. There should be particular instruction in assiduous sterile technique and emphasis on obtaining simultaneous paired specimens from two different anatomic sites. Whenever it has been evaluated, professional blood drawing teams always have a lower rate of blood culture contamination than do nonprofessionals (students, housestaff, and nurses). ${ }^{6} \mathrm{~A}$ calculation of the money saved by not having to process contaminated specimens would probably reveal that professional blood culture teams pay for themselves. There would be little educational value lost if students and housestaff no longer performed these procedures.

If current trends are an indication, coagulase-negative staphylococci will continue to increase in importance as nosocomial pathogens. ${ }^{5}$ Thus, the dilemma over the significance of a "positive" culture for these organisms will arise more frequently. The importance of the clinician's need to have rapid susceptibility data at his fingertips in specific situations will have to be balanced by the increasing economic constraints of the clinical microbiology laboratory. Active, ongoing cooperation between the two will become increasingly critical.

\section{REFERENCES}

1. Kirchhoff LV. Sheagren JN: Epidemiology and clinical significance of blood cultures positive for coagulase-negative staphylococous. Infed Comtrol 1985: $6(12): 479-486$.

2. Weinstein MP. Reller LB. Murphy JR, et al: The clinical significance of positive blood cultures: $A$ comprehensive analysis of 500 episodes of bacteremia and fungemia in adults. 1. Laboratory and epidemiologic observations. Rev Infect Dis 1983: 5:35-53.

3. Christensen (GD, Parisi JT, Bisno AL. et al: Characterization of clinically significant strains of coagulase-negative staphylococci. J Clin Mirrobiol 1983: 18:258-269

4. Archer GL. Karchmer AW. Vishniarsky N, et al: Plasmid-pattern analysis for the differentiation of infecting from noninfecting Staphylococcus epidermidis. I Infert Dis 1984; 149:913-920.

5. Ponce de Leon S. Wenzel RP: Hospital-acquired bloodstream infections with Staphylorocrus epidermidis: Review of 100 cases. Am / Med 1984: 77:639-644

6. Washington JA: Conventional approaches 10 blood culare, in Washington JA (ed): The Detection of Septicemin. West Palm Beach, CRC l'ress, 1978. pp 41-88. 